\title{
The Meir-Keeler Type for Solving Variational Inequalities and Fixed Points of Nonexpansive Semigroups in Banach Spaces
}

\author{
Phayap Katchang $^{1,2}$ and Poom Kumam ${ }^{2}$ \\ ${ }^{1}$ Department of Mathematics and Statistics, Faculty of Science and Agricultural Technology, Rajamangala \\ University of Technology Lanna Tak, Tak 63000, Thailand \\ ${ }^{2}$ Department of Mathematics, Faculty of Science, King Mongkut's University of Technology Thonburi \\ (KMUTT), Bang Mod, Thrung Khru, Bangkok 10140, Thailand
}

Correspondence should be addressed to Poom Kumam, poom.kum@kmutt.ac.th

Received 7 June 2012; Revised 19 August 2012; Accepted 27 August 2012

Academic Editor: Juan Torregrosa

Copyright (c) 2012 P. Katchang and P. Kumam. This is an open access article distributed under the Creative Commons Attribution License, which permits unrestricted use, distribution, and reproduction in any medium, provided the original work is properly cited.

The aim of this paper is to introduce a new iterative scheme for finding common solutions of the variational inequalities for an inverse strongly accretive mapping and the solutions of fixed point problems for nonexpansive semigroups by using the modified viscosity approximation method associate with Meir-Keeler type mappings and obtain some strong convergence theorem in a Banach spaces under some parameters controlling conditions. Our results extend and improve the recent results of Li and Gu (2010), Wangkeeree and Preechasilp (2012), Yao and Maruster (2011), and many others.

\section{Introduction}

The theory of variational inequalities and variational inclusions are among the most interesting and important mathematical problems and have been studied intensively in the past years since they have wide applications in the optimization and control, economics, engineering science, physical sciences, and applied sciences. For these reasons, many existence result and iterative algorithms for various variational inclusion have been studied extensively by many authors (see, e.g., [1-8]). The important generalization of variational inequalities has been extensively studied and generalized in different directions to study a wide class of problems arising in mechanics, optimization, nonlinear programming, finance, and applied sciences (see, e.g., $[2,8]$ ). 
Let $C$ be a nonempty closed convex subset of a real Banach space $E$ and $E^{*}$ be the dual space of $E$ with norm $\|\cdot\|$ and $\langle\cdot, \cdot\rangle$ pairing between $E$ and $E^{*}$. For $q>1$, the generalized duality mapping $J_{q}: E \rightarrow 2^{E^{*}}$ is defined by

$$
J_{q}(x)=\left\{f \in E^{*}:\langle x, f\rangle=\|x\|^{q},\|f\|=\|x\|^{q-1}\right\}
$$

for all $x \in E$. In particular, if $q=2$, the mapping $J_{2}$ is called the normalized duality mapping and, usually, written $J_{2}=J$. Further, we have the following properties of the generalized duality mapping $J_{q}$ : (i) $J_{q}(x)=\|x\|^{q-2} J_{2}(x)$ for all $x \in E$ with $x \neq 0$; (ii) $J_{q}(t x)=t^{q-1} J_{q}(x)$ for all $x \in E$ and $t \in[0, \infty)$; (iii) $J_{q}(-x)=-J_{q}(x)$ for all $x \in E$.

Recall that a mapping $A: C \rightarrow C$ is said to be (i) Lipschitzian with Lipschitz constant $L>0$ if $\|A x-A y\| \leq L\|x-y\|$, for all $x, y \in C$; (ii) contraction if there exists a constant $\alpha \in(0,1)$ such that $\|A x-A y\| \leq \alpha\|x-y\|$, for all $x, y \in C$; (iii) nonexpansive if $\|A x-A y\| \leq\|x-y\|$, for all $x, y \in C$. An operator $A: C \rightarrow E$ is said to be

(i) accretive if there exists $j(x-y) \in J(x-y)$ such that

$$
\langle A x-A y, j(x-y)\rangle \geq 0, \quad \forall x, y \in C,
$$

(ii) $\beta$-strongly accretive if there exists a constant $\beta>0$ such that

$$
\langle A x-A y, j(x-y)\rangle \geq \beta\|x-y\|^{2}, \quad \forall x, y \in C,
$$

(iii) $\beta$-inverse strongly accretive if, for any $\beta>0$,

$$
\langle A x-A y, j(x-y)\rangle \geq \beta\|A x-A y\|^{2}, \quad \forall x, y \in C .
$$

Let $D$ be a subset of $C$ and $Q: C \rightarrow D$. Then $Q$ is said to sunny if $Q(Q x+t(x-Q x))=$ $Q x$, whenever $Q x+t(x-Q x) \in C$ for $x \in C$ and $t \geq 0$. A subset $D$ of $C$ is said to be a sunny nonexpansive retract of $C$ if there exists a sunny nonexpansive retraction $Q$ of $C$ onto $D$. A mapping $Q: C \rightarrow C$ is called a retraction if $Q^{2}=Q$. If a mapping $Q: C \rightarrow C$ is a retraction, then $Q z=z$ for all $z$ is in the range of $Q$.

A family $\mathcal{S}=\{T(t): 0 \leq t<\infty\}$ of mappings of $C$ into itself is called a nonexpansive semigroup on $C$ if it satisfies the following conditions:

(i) $T(0) x=x$ for all $x \in C$;

(ii) $T(s+t)=T(s) T(t)$ for all $s, t \geq 0$;

(iii) $\|T(t) x-T(t) y\| \leq\|x-y\|$ for all $x, y \in C$ and $t \geq 0$;

(iv) for all $x \in C, t \mapsto T(t) x$ is continuous.

We denote by $F(\mathcal{S})$ the set of all common fixed points of $\mathcal{S}=\{T(t): 0 \leq t<$ $\infty\}$, that is, $F(\mathcal{S})=\cap_{t \geq 0} F(T(t))$. It is known that $F(\mathcal{S})$ is closed and convex (see also $[9,10]$ ).

A mapping $\psi: \mathbb{R}^{+} \rightarrow \mathbb{R}^{+}$is said to be an $L$ function if $\psi(0)=0, \psi(t)>0$ for each $t>0$, and for every $s>0$ there exists $u>s$ such that $\psi(t) \leq s$ for all $t \in[s, u]$. As a consequence, every $L$-function $\psi$ satisfies $\psi(t)<t$ for each $t>0$. 
Definition 1.1. Let $(X, d)$ be a matric space. A mapping $f: X \rightarrow X$ is said to be:

(i) $(\psi, L)$-contraction if $\psi: \mathbb{R}^{+} \rightarrow \mathbb{R}^{+}$is an $L$-function and $d(f(x), f(y))<\psi(d(x, y))$ for all $x, y \in X$ with $x \neq y$;

(ii) Meir-Keeler type mapping if for each $\epsilon>0$ there exists $\delta=\delta(\epsilon)>0$ such that for each $x, y \in X$ with $d(x, y)<\epsilon+\delta$ we have $d(f(x), f(y))<\epsilon$.

Remark 1.2. From Definition 1.1, if $\psi(t)=\alpha t, \alpha \in[0,1), t \in \mathbb{R}^{+}$, then we get the usual contraction mapping with coefficient $\alpha$.

At the same time, we are also interesting in the variational inequality problems for an inverse strongly accretive mappings in Banach spaces. In 2006, Aoyama et al. [11] introduced the following iteration scheme for an inverse strongly accretive operator $A$ in Banach spaces E:

$$
\begin{gathered}
x_{1}=x \in C, \\
x_{n+1}=\alpha_{n} x_{n}+\left(1-\alpha_{n}\right) Q_{C}\left(x_{n}-\lambda_{n} A x_{n}\right),
\end{gathered}
$$

for all $n \geq 1$, where $C \subset E$ and $Q_{C}$ is a sunny nonexpansive retraction from $E$ onto $C$. They proved a weak convergence theorem in a Banach spaces. Moreover, the sequence $\left\{x_{n}\right\}$ in (1.5) solved the generalized variational inequality problem for finding a point $x \in C$ such that

$$
\langle A x, j(y-x)\rangle \geq 0
$$

for all $y \in C$. The set of solutions of (1.6) is denoted by $V I(C, A)$.

An interesting is the proof by using a nonexpansive semigroup and Meir-Keeler type mapping, in 2010, $\mathrm{Li}$ and $\mathrm{Gu}$ [12] defined the following sequence:

$$
\begin{gathered}
x_{1}=x \in E, \\
y_{n}-\alpha_{n} x_{n}+\left(1-\alpha_{n}\right) T\left(t_{n}\right) x_{n}, \\
x_{n+1}=\beta_{n} f\left(x_{n}\right)+\left(1-\beta_{n}\right) y_{n}, \quad n \geq 1 .
\end{gathered}
$$

Wangkeeree and Preechasilp [13] introduced the following iterative scheme:

$$
\begin{gathered}
x_{0} \in C, \\
z_{n}-\gamma_{n} x_{n}+\left(1-\gamma_{n}\right) T\left(t_{n}\right) x_{n}, \\
y_{n}-\alpha_{n} x_{n}+\left(1-\alpha_{n}\right) T\left(t_{n}\right) z_{n}, \\
x_{n+1}=\beta_{n} f\left(x_{n}\right)+\left(1-\beta_{n}\right) y_{n}, \quad n \geq 0 .
\end{gathered}
$$


In 2011, Yao and Maruster [8] proved some strong convergence theorems for finding a solution of variational inequality problem (1.6) in Banach spaces. They defined a sequence $\left\{x_{n}\right\}$ iteratively by given arbitrarily $x_{0} \in C$ and

$$
x_{n+1}=\beta_{n} x_{n}+\left(1-\beta_{n}\right) Q_{C}\left[\left(1-\alpha_{n}\right)\left(x_{n}-\lambda A x_{n}\right)\right], \quad \forall n \geq 0,
$$

where $Q_{C}$ is a sunny nonexpansive retraction from a uniformly convex and 2-uniformly smooth Banach space $E$, and $A$ is an $\alpha$-inverse strongly accretive operator of $C$ into $E$.

Motivated and inspired by the idea of Li and Gu [12], Wangkeeree and Preechasilp [13], and Yao and Maruster [8], in this paper, we introduce a new iterative scheme for finding common solutions of the variational inequalities for an inverse strongly accretive mapping and the solutions of fixed point problems for a nonexpansive semigroup by using the modified viscosity approximation method associated with Meir-Keeler type mapping. We will prove the strong convergence theorem under some parameters controlling conditions. Our results extend and improve the recent results of $\mathrm{Li}$ and $\mathrm{Gu}$ [12], Wangkeeree and Preechasilp [13], Yao and Maruster [8], and many others.

\section{Preliminaries}

Let $U=\{x \in E:\|x\|=1\}$. A Banach space $E$ is said to uniformly convex if, for any $\epsilon \in(0,2]$, there exists $\delta>0$ such that, for any $x, y \in U,\|x-y\| \geq \epsilon$ implies $\|(x+y) / 2\| \leq 1-\delta$. It is known that a uniformly convex Banach space is reflexive and strictly convex. A Banach space $E$ is said to be smooth if the $\operatorname{limit}_{\lim _{t \rightarrow 0}}((\|x+t y\|-\|x\|) / t)$ exists for all $x, y \in U$. It is also said to be uniformly smooth if the limit is attained uniformly for $x, y \in U$. The modulus of smoothness of $E$ is defined by

$$
\rho(\tau)=\sup \left\{\frac{1}{2}(\|x+y\|+\|x-y\|)-1: x, y \in E,\|x\|=1,\|y\|=\tau\right\}
$$

where $\rho:[0, \infty) \rightarrow[0, \infty)$ is a function. It is known that $E$ is uniformly smooth if and only if $\lim _{\tau \rightarrow 0}(\rho(\tau) / \tau)=0$. Let $q$ be a fixed real number with $1<q \leq 2$. A Banach space $E$ is said to be $q$-uniformly smooth if there exists a constant $c>0$ such that $\rho(\tau) \leq c \tau^{q}$ for all $\tau>0$.

A Banach space $E$ is said to satisfy Opial's condition if for any sequence $\left\{x_{n}\right\}$ in $E$, $x_{n} \rightarrow x(n \rightarrow \infty)$ implies that

$$
\limsup _{n \rightarrow \infty}\left\|x_{n}-x\right\|<\limsup _{n \rightarrow \infty}\left\|x_{n}-y\right\|, \quad \forall y \in E \text { with } x \neq y
$$

By [14, Theorem 1], it is well known that if $E$ admits a weakly sequentially continuous duality mapping, then $E$ satisfies Opial's condition, and $E$ is smooth.

The following result describes a characterization of sunny nonexpansive retractions on a smooth Banach space. 
Proposition 2.1 (see [15]). Let $E$ be a smooth Banach space and let $C$ be a nonempty subset of $E$. Let $Q: E \rightarrow C$ be a retraction, and let $J$ be the normalized duality mapping on $E$. Then the following are equivalent:

(i) $Q$ is sunny and nonexpansive;

(ii) $\|Q x-Q y\|^{2} \leq\langle x-y, J(Q x-Q y)\rangle, \forall x, y \in E$;

(iii) $\langle x-Q x, J(y-Q x)\rangle \leq 0, \forall x \in E, y \in C$.

Proposition 2.2 (see [16]). Let $C$ be a nonempty closed convex subset of a uniformly convex and uniformly smooth Banach space $E$, and let $T$ be a nonexpansive mapping of $C$ into itself with $F(T) \neq \emptyset$. Then the set $F(T)$ is a sunny nonexpansive retract of $C$.

Lemma 2.3 (see [17]). Let $C$ be a nonempty bounded closed convex subset of a uniformly convex Banach space $E$ and $T: C \rightarrow C$ be a nonexpansive mapping. If $\left\{x_{n}\right\}$ is a sequence of $C$ such that $x_{n} \rightarrow x$ and $x_{n}-T x_{n} \rightarrow 0$, then $x$ is a fixed point of $T$.

We need the following lemmas for proving our main results.

Lemma 2.4 (see [18]). Let $r>0$, and let E be a uniformly convex Banach space. Then, there exists a continuous, strictly increasing, and convex function $g:[0, \infty) \rightarrow[0, \infty)$ with $g(0)=0$ such that

$$
\|\lambda x+(1-\lambda) y\|^{2} \leq \lambda\|x\|^{2}+(1-\lambda)\|y\|^{2}-\lambda(1-\lambda) g(\|x-y\|)
$$

for all $x, y \in B_{r}:=\{z \in E:\|z\| \leq r\}$ and $0 \leq \lambda \leq 1$.

Lemma 2.5 (see [19]). Let $E$ be a real smooth and uniformly convex Banach space, and let $r>0$. Then there exists a strictly increasing, continuous, and convex function $g:[0,2 r] \rightarrow \mathbb{R}$ such that $g(0)=0$ and

$$
g(\|x-y\|) \leq\|x\|^{2}-2\langle x, j y\rangle+\|y\|^{2}, \quad \forall x, y \in B_{r} .
$$

Lemma 2.6 (see [18]). Let E be a real 2-uniformly smooth Banach space with the best smooth constant $K$. Then the following inquality holds:

$$
\|x+y\|^{2} \leq\|x\|^{2}+2\langle y, J x\rangle+2\|K y\|^{2}, \quad \forall x, y \in E .
$$

Lemma 2.7 (see [20]). Let $E$ be a real Banach space and $J: E \rightarrow 2^{E^{*}}$ be the normalized duality mapping. Then, for any $x, y \in E$, one has

$$
\|x+y\|^{2} \leq\|x\|^{2}+2\langle y, J(x+y)\rangle
$$

for all $j(x+y) \in J(x+y)$ with $x \neq y$.

Lemma 2.8 (see [21]). Let $\left\{x_{n}\right\}$ and $\left\{y_{n}\right\}$ be bounded sequences in a Banach space $X$, and let $\left\{\beta_{n}\right\}$ be a sequence in $[0,1]$ with $0<\liminf _{n \rightarrow \infty} \beta_{n} \leq \limsup _{n \rightarrow \infty} \beta_{n}<1$. Suppose that $x_{n+1}=\left(1-\beta_{n}\right) y_{n}+$ $\beta_{n} x_{n}$ for all integers $n \geq 0$ and $\lim \sup _{n \rightarrow \infty}\left(\left\|y_{n+1}-y_{n}\right\|-\left\|x_{n+1}-x_{n}\right\|\right) \leq 0$. Then, $\lim _{n \rightarrow \infty}\left\|y_{n}-x_{n}\right\|=$ 0 . 
Lemma 2.9 (see [22]). Assume that $\left\{a_{n}\right\}$ is a sequence of nonnegative real numbers such that

$$
a_{n+1} \leq\left(1-\alpha_{n}\right) a_{n}+\delta_{n}, \quad n \geq 0
$$

where $\left\{\alpha_{n}\right\}$ is a sequence in $(0,1)$, and $\left\{\delta_{n}\right\}$ is a sequence in $\mathbb{R}$ such that

(1) $\sum_{n=1}^{\infty} \alpha_{n}=\infty$

(2) $\lim \sup _{n \rightarrow \infty}\left(\delta_{n} / \alpha_{n}\right) \leq 0$ or $\sum_{n=1}^{\infty}\left|\delta_{n}\right|<\infty$.

Then $\lim _{n \rightarrow \infty} a_{n}=0$.

Theorem 2.10 (see [23]). Let $(X, d)$ be a complete metric space and $f: X \rightarrow X$ a Meir-Keeler type mapping. Then $f$ has a unique fixed point.

Theorem 2.11 (see [24]). Let $(X, d)$ be a metric space and $f: X \rightarrow X$ a mapping. Then the following assertions are equivalent:

(i) $f$ is a Meir-Keeler type mapping;

(ii) there exists an Lunction $\psi: \mathbb{R}^{+} \rightarrow \mathbb{R}^{+}$such that $f$ is a $(\psi, L)$ contraction.

Proposition 2.12 (see [21]). Let $C$ be a convex subset of a Banach space E. Let $f: C \rightarrow C$ be a Meir-Keeler type mapping. Then for each $\epsilon>0$ there exists $r \in(0,1)$ such that for each $x, y \in C$ with $\|x-y\| \geq \epsilon$, one has

$$
\|f(x)-f(y)\| \leq r\|x-y\| .
$$

Proposition 2.13 (see [21]). Let $C$ be a convex subset of a Banach space E. Let $T$ be a nonexpansive mapping on $C$, and let $f: C \rightarrow C$ be a Meir-Keeler-type mapping. Then the following holds:

(i) $T \circ f$ is a Meir-Keeler type mapping on $C$;

(ii) for each $\alpha \in(0,1)$ the mapping $x \mapsto \alpha f(x)+(1-\alpha) T(x)$ is a Meir-Keeler-type mapping on $C$.

The following lemma is characterized by the set of solutions of variational inequality by using sunny nonexpansive retractions.

Lemma 2.14 (see [11]). Let $C$ be a nonempty closed convex subset of a smooth Banach space E. Let $Q_{C}$ be a sunny nonexpansive retraction from $E$ onto $C$, and let $A$ be an accretive operator of $C$ into $E$. Then, for all $\lambda>0$,

$$
V I(C, A)=F(Q(I-\lambda A))
$$

where $\operatorname{VI}(C, A)=\left\{x^{*} \in C:\left\langle A x^{*}, J\left(x-x^{*}\right)\right\rangle \geq 0, \forall x \in C\right\}$.

\section{Strong Convergence Theorems}

In this section, we suppose that the function $\psi$ from the definition of the $(\psi, L)$ contraction is continuous and strictly increasing and $\lim _{t \rightarrow \infty} \eta(t)=\infty$, where $\eta(t)=t-\psi(t), t \in \mathbb{R}^{+}$. 
In consequence, we have that $\eta$ is a bijection on $\mathbb{R}^{+}$and the function $\psi$ satisfies the assumption in Remark 1.2. conditions:

Suppose that $\left\{\alpha_{n}\right\},\left\{\beta_{n}\right\} \subset(0,1)$, and $\left\{\mu_{n}\right\},\left\{\lambda_{n}\right\} \subset(0, \infty)$ satisfy the following

(C1) $\lim _{n \rightarrow \infty} \alpha_{n}=0$ and $\sum_{n=0}^{\infty} \alpha_{n}=\infty$;

(C2) $\lim _{n \rightarrow \infty}\left|\lambda_{n+1}-\lambda_{n}\right|=0$ and $0<a \leq \lambda_{n} \leq b<\beta / K^{2}$;

(C3) $0<\liminf _{n \rightarrow \infty} \beta_{n} \leq \lim \sup _{n \rightarrow \infty} \beta_{n}<1$;

(C4) $\lim _{n \rightarrow \infty} \mu_{n}=0$;

(C5) $\lim _{n \rightarrow \infty} \sup _{x \in \tilde{C}}\left\|T\left(\mu_{n+1}\right) x-T\left(\mu_{n}\right) x\right\|=0, \widetilde{C}$ bounded subset of $C$.

Next, we stat the main result.

Theorem 3.1. Let E be a uniformly convex and 2-uniformly smooth Banach space with the best smooth constant $K$ and $C$ a nonempty closed convex subset of $E$. Let $Q_{C}$ be a sunny nonexpansive retraction from $E$ onto $C$ and $A: C \rightarrow E$ be an $\beta$-inverse-strongly accretive operator. Let $\mathcal{S}=\{T(t): t \geq 0\}$ be a nonexpansive semigroup from $C$ into itself and $f$ be a Meir-Keeler contraction of $C$ into itself. Suppose that $F:=F(S) \cap V I(C, A) \neq \emptyset$ and the conditions (C1)-(C5). For arbitrary given $x_{1} \in C$, the sequences $\left\{x_{n}\right\}$ are generated by

$$
\begin{gathered}
u_{n}=Q_{C}\left(x_{n}-\lambda_{n} A x_{n}\right), \\
y_{n}=Q_{C}\left[\alpha_{n} f\left(x_{n}\right)+\left(1-\alpha_{n}\right) u_{n}\right], \\
x_{n+1}=\beta_{n} x_{n}+\left(1-\beta_{n}\right) T\left(\mu_{n}\right) y_{n} .
\end{gathered}
$$

Then $\left\{x_{n}\right\}$ converges strongly to $x^{*}=Q_{F} f x^{*} \in F$ which also solves the following variational inequality:

$$
\left\langle(f-I) x^{*}, J\left(z-x^{*}\right)\right\rangle \leq 0, \quad \forall z \in F
$$

Proof. First we prove that $\left\{x_{n}\right\}$ bounded. Let $p \in F$, we have

$$
\begin{aligned}
\left\|u_{n}-p\right\|^{2} & =\left\|Q_{C}\left(x_{n}-\lambda_{n} A x_{n}\right)-Q_{C}\left(p-\lambda_{n} A p\right)\right\|^{2} \\
& \leq\left\|\left(x_{n}-\lambda_{n} A x_{n}\right)-\left(p-\lambda_{n} A p\right)\right\|^{2} \\
& =\left\|\left(I-\lambda_{n} A\right) x_{n}-\left(I-\lambda_{n} A\right) p\right\|^{2} \\
& =\left\|\left(x_{n}-p\right)-\lambda_{n}\left(A x_{n}-A p\right)\right\|^{2} \\
& \leq\left\|x_{n}-p\right\|^{2}-2 \lambda_{n}\left\langle A x_{n}-A p, J\left(x_{n}-p\right)\right\rangle+2 K^{2} \lambda_{n}^{2}\left\|A x_{n}-A p\right\|^{2} \\
& \leq\left\|x_{n}-p\right\|^{2}-2 \lambda_{n} \beta\left\|A x_{n}-A p\right\|^{2}+2 K^{2} \lambda_{n}^{2}\left\|A x_{n}-A p\right\|^{2} \\
& =\left\|x_{n}-p\right\|^{2}+2 \lambda_{n}\left(\lambda_{n} K^{2}-\beta\right)\|A x-A y\|^{2} \\
& \leq\left\|x_{n}-p\right\|^{2} .
\end{aligned}
$$


So, we get $\left\|u_{n}-p\right\| \leq\left\|x_{n}-p\right\|$, for all $n \geq 1$. It follows that

$$
\begin{aligned}
\left\|x_{n+1}-p\right\| & =\left\|\beta_{n}\left(x_{n}-p\right)+\left(1-\beta_{n}\right)\left(T\left(\mu_{n}\right) y_{n}-p\right)\right\| \\
& \leq \beta_{n}\left\|x_{n}-p\right\|+\left(1-\beta_{n}\right)\left\|y_{n}-p\right\| \\
& =\beta_{n}\left\|x_{n}-p\right\|+\left(1-\beta_{n}\right)\left\|Q_{C}\left[\alpha_{n} f\left(x_{n}\right)+\left(1-\alpha_{n}\right) u_{n}\right]-Q_{C} p\right\| \\
& \leq \beta_{n}\left\|x_{n}-p\right\|+\left(1-\beta_{n}\right)\left[\alpha_{n}\left\|f\left(x_{n}\right)-p\right\|+\left(1-\alpha_{n}\right)\left\|u_{n}-p\right\|\right] \\
& \leq \beta_{n}\left\|x_{n}-p\right\|+\left(1-\beta_{n}\right)\left[\alpha_{n}\left\|f\left(x_{n}\right)-f(p)\right\|+\alpha_{n}\|f(p)-p\|+\left(1-\alpha_{n}\right)\left\|x_{n}-p\right\|\right] \\
& \leq \beta_{n}\left\|x_{n}-p\right\|+\left(1-\beta_{n}\right)\left[\alpha_{n} \psi\left(\left\|x_{n}-p\right\|\right)+\alpha_{n}\|f(p)-p\|+\left(1-\alpha_{n}\right)\left\|x_{n}-p\right\|\right] \\
& =\left\|x_{n}-p\right\|+\alpha_{n}\left(1-\beta_{n}\right)\left[\eta\left(\left\|x_{n}-p\right\|\right)\right]+\alpha_{n}\left(1-\beta_{n}\right) \eta\left[\eta^{-1}(\|f(p)-p\|)\right] \\
& \leq \max \left\{\left\|x_{n}-p\right\|, \eta^{-1}(\|f(p)-p\|)\right\} .
\end{aligned}
$$

By induction, we conclude that

$$
\left\|x_{n}-p\right\| \leq \max \left\{\left\|x_{n}-p\right\|, \eta^{-1}(\|f(p)-p\|)\right\}, \quad \forall n \geq 1
$$

This implies that $\left\{x_{n}\right\}$ bounded, so are $\left\{f\left(x_{n}\right)\right\},\left\{y_{n}\right\},\left\{u_{n}\right\},\left\{A x_{n}\right\}$, and $\left\{T\left(\mu_{n}\right) y_{n}\right\}$.

Next, we show that $\lim _{n \rightarrow \infty}\left\|x_{n+1}-x_{n}\right\|=0$, we observe that

$$
\begin{aligned}
\left\|u_{n+1}-u_{n}\right\|= & \left\|Q_{C}\left(x_{n+1}-\lambda_{n+1} A x_{n+1}\right)-Q_{C}\left(x_{n}-\lambda_{n} A x_{n}\right)\right\| \\
\leq & \left\|\left(x_{n+1}-\lambda_{n+1} A x_{n+1}\right)-\left(x_{n}-\lambda_{n} A x_{n}\right)\right\| \\
& =\left\|\left(x_{n+1}-\lambda_{n+1} A x_{n+1}\right)-\left(x_{n}-\lambda_{n+1} A x_{n}\right)+\left(\lambda_{n+1}-\lambda_{n}\right) A x_{n}\right\| \\
\leq & \left\|\left(I-\lambda_{n+1} A\right) x_{n+1}-\left(I-\lambda_{n+1} A\right) x_{n}\right\|+\left|\lambda_{n+1}-\lambda_{n}\right|\left\|A x_{n}\right\| \\
\leq & \left\|x_{n+1}-x_{n}\right\|+\mid \lambda_{n+1}-\lambda_{n}\left\|A x_{n}\right\|, \\
\left\|y_{n+1}-y_{n}\right\|= & \left\|Q_{C}\left[\alpha_{n+1} f\left(x_{n+1}\right)+\left(1-\alpha_{n+1}\right) u_{n+1}\right]-Q_{C}\left[\alpha_{n} f\left(x_{n}\right)+\left(1-\alpha_{n}\right) u_{n}\right]\right\| \\
\leq & \left\|\left[\alpha_{n+1} f\left(x_{n+1}\right)+\left(1-\alpha_{n+1}\right) u_{n+1}\right]-\left[\alpha_{n} f\left(x_{n}\right)+\left(1-\alpha_{n}\right) u_{n}\right]\right\| \\
\leq & \left\|u_{n+1}-u_{n}\right\|+\alpha_{n+1}\left(\left\|u_{n+1}\right\|+\left\|f\left(x_{n+1}\right)\right\|\right)+\alpha_{n}\left(\left\|u_{n}\right\|+\left\|f\left(x_{n}\right)\right\|\right) \\
\leq & \left\|x_{n+1}-x_{n}\right\|+\mid \lambda_{n+1}-\lambda_{n}\left\|A x_{n}\right\|+\alpha_{n+1}\left(\left\|u_{n+1}\right\|+\left\|f\left(x_{n+1}\right)\right\|\right) \\
& +\alpha_{n}\left(\left\|u_{n}\right\|+\left\|f\left(x_{n}\right)\right\|\right) .
\end{aligned}
$$


It follows that

$$
\begin{aligned}
\left\|T\left(\mu_{n+1}\right) y_{n+1}-T\left(\mu_{n}\right) y_{n}\right\| \leq & \left\|T\left(\mu_{n+1}\right) y_{n+1}-T\left(\mu_{n+1}\right) y_{n}\right\|+\left\|T\left(\mu_{n+1}\right) y_{n}-T\left(\mu_{n}\right) y_{n}\right\| \\
\leq & \left\|y_{n+1}-y_{n}\right\|+\left\|T\left(\mu_{n+1}\right) y_{n}-T\left(\mu_{n}\right) y_{n}\right\| \\
\leq & \left\|x_{n+1}-x_{n}\right\|+\mid \lambda_{n+1}-\lambda_{n}\left\|A x_{n}\right\|+\alpha_{n+1}\left(\left\|u_{n+1}\right\|+\left\|f\left(x_{n+1}\right)\right\|\right) \\
& +\alpha_{n}\left(\left\|u_{n}\right\|+\left\|f\left(x_{n}\right)\right\|\right)+\left\|T\left(\mu_{n+1}\right) y_{n}-T\left(\mu_{n}\right) y_{n}\right\| \\
\leq & \left\|x_{n+1}-x_{n}\right\|+\mid \lambda_{n+1}-\lambda_{n}\left\|A x_{n}\right\|+\alpha_{n+1}\left(\left\|u_{n+1}\right\|+\left\|f\left(x_{n+1}\right)\right\|\right) \\
& +\alpha_{n}\left(\left\|u_{n}\right\|+\left\|f\left(x_{n}\right)\right\|\right)+\sup _{y \in\left\{y_{n}\right\}}\left\|T\left(\mu_{n+1}\right) y-T\left(\mu_{n}\right) y\right\| .
\end{aligned}
$$

By (C1), (C2), and (C4), they imply that

$$
\limsup _{n \rightarrow \infty}\left(\left\|T\left(\mu_{n+1}\right) y_{n+1}-T\left(\mu_{n}\right) y_{n}\right\|-\left\|x_{n+1}-x_{n}\right\|\right) \leq 0
$$

Applying Lemma 2.8, we obtain

$$
\lim _{n \rightarrow \infty}\left\|T\left(\mu_{n}\right) y_{n}-x_{n}\right\|=0
$$

Therefore, we have

$$
\lim _{n \rightarrow \infty}\left\|x_{n+1}-x_{n}\right\|=0
$$

On the other hand, we consider

$$
\begin{aligned}
\left\|x_{n+1}-p\right\|^{2} \leq & \beta_{n}\left\|x_{n}-p\right\|^{2}+\left(1-\beta_{n}\right)\left\|y_{n}-p\right\|^{2} \\
\leq & \beta_{n}\left\|x_{n}-p\right\|^{2}+\left(1-\beta_{n}\right)\left[\alpha_{n}\left\|f\left(x_{n}\right)-p\right\|^{2}+\left(1-\alpha_{n}\right)\left\|u_{n}-p\right\|^{2}\right] \\
= & \beta_{n}\left\|x_{n}-p\right\|^{2}+\left(1-\beta_{n}\right) \\
& \times\left[\alpha_{n}\left\|f\left(x_{n}\right)-p\right\|^{2}+\left(1-\alpha_{n}\right)\left\|Q_{C}\left(x_{n}-\lambda_{n} A x_{n}\right)-Q_{C}\left(p-\lambda_{n} A p\right)\right\|^{2}\right] \\
\leq & \beta_{n}\left\|x_{n}-p\right\|^{2}+\left(1-\beta_{n}\right) \\
& \times\left[\alpha_{n}\left\|f\left(x_{n}\right)-p\right\|^{2}+\left(1-\alpha_{n}\right)\left(\left\|x_{n}-p\right\|^{2}+2 \lambda_{n}\left(\lambda_{n} K^{2}-\beta\right)\|A x-A y\|^{2}\right)\right] \\
= & {\left[\beta_{n}+\left(1-\beta_{n}\right)\left(1-\alpha_{n}\right)\right]\left\|x_{n}-p\right\|^{2}+\alpha_{n}\left(1-\beta_{n}\right)\left\|f\left(x_{n}\right)-p\right\|^{2} } \\
& +2 \lambda_{n}\left(\lambda_{n} K^{2}-\beta\right)\left(1-\beta_{n}\right)\left(1-\alpha_{n}\right)\|A x-A y\|^{2} \\
\leq & \left\|x_{n}-p\right\|^{2}+\alpha_{n}\left\|f\left(x_{n}\right)-p\right\|^{2}+2 \lambda_{n}\left(\lambda_{n} K^{2}-\beta\right)\|A x-A y\|^{2} .
\end{aligned}
$$


Then, we obtain that

$$
\begin{aligned}
2 \lambda_{n}\left(\beta-\lambda_{n} K^{2}\right)\|A x-A y\|^{2} & \leq\left\|x_{n}-p\right\|^{2}-\left\|x_{n+1}-p\right\|^{2}+\alpha_{n}\left\|f\left(x_{n}\right)-p\right\|^{2} \\
& \leq\left\|x_{n}-x_{n+1}\right\|\left(\left\|x_{n}-p\right\|+\left\|x_{n+1}-p\right\|\right)+\alpha_{n}\left\|f\left(x_{n}\right)-p\right\|^{2} .
\end{aligned}
$$

By (C1), (C2), (C3), and (3.10), we get

$$
\lim _{n \rightarrow \infty}\left\|A x_{n}-A p\right\|=0
$$

From Proposition 2.1 (ii) and Lemma 2.5, we also have

$$
\begin{aligned}
\left\|u_{n}-p\right\|^{2} & =\left\|Q_{C}\left(x_{n}-\lambda_{n} A x_{n}\right)-Q_{C}\left(p-\lambda_{n} A p\right)\right\|^{2} \\
& \leq\left\langle\left(x_{n}-\lambda_{n} A x_{n}\right)-\left(p-\lambda_{n} A p\right), J\left(u_{n}-p\right)\right\rangle \\
& =\left\langle\left(x_{n}-p\right)-\lambda_{n}\left(A x_{n}-A p\right), J\left(u_{n}-p\right)\right\rangle \\
& =\left\langle x_{n}-p, J\left(u_{n}-p\right)\right\rangle-\lambda_{n}\left\langle A x_{n}-A p, J\left(u_{n}-p\right)\right\rangle \\
& \leq \frac{1}{2}\left[\left\|x_{n}-p\right\|^{2}+\left\|u_{n}-p\right\|^{2}-g\left\|x_{n}-u_{n}\right\|\right]+\lambda_{n}\left\|A x_{n}-A p\right\|\left\|u_{n}-p\right\| .
\end{aligned}
$$

So, we get,

$$
\left\|u_{n}-p\right\|^{2} \leq\left\|x_{n}-p\right\|^{2}-g\left\|x_{n}-u_{n}\right\|+2 \lambda_{n}\left\|A x_{n}-A p\right\|\left\|u_{n}-p\right\| .
$$

Therefore, using (3.11), we obtain

$$
\begin{aligned}
\left\|x_{n+1}-p\right\|^{2} \leq & \beta_{n}\left\|x_{n}-p\right\|^{2}+\left(1-\beta_{n}\right)\left[\alpha_{n}\left\|f\left(x_{n}\right)-p\right\|^{2}+\left(1-\alpha_{n}\right)\left\|u_{n}-p\right\|^{2}\right] \\
= & \beta_{n}\left\|x_{n}-p\right\|^{2}+\alpha_{n}\left(1-\beta_{n}\right)\left\|f\left(x_{n}\right)-p\right\|^{2}+\left(1-\alpha_{n}\right)\left(1-\beta_{n}\right)\left\|u_{n}-p\right\|^{2} \\
\leq & \beta_{n}\left\|x_{n}-p\right\|^{2}+\alpha_{n}\left(1-\beta_{n}\right)\left\|f\left(x_{n}\right)-p\right\|^{2} \\
& +\left(1-\alpha_{n}\right)\left(1-\beta_{n}\right)\left[\left\|x_{n}-p\right\|^{2}-g\left(\left\|x_{n}-u_{n}\right\|\right)+2 \lambda_{n}\left\|A x_{n}-A p\right\|\left\|u_{n}-p\right\|\right] \\
\leq & \left\|x_{n}-p\right\|^{2}+\alpha_{n}\left\|f\left(x_{n}\right)-p\right\|^{2}-g\left(\left\|x_{n}-u_{n}\right\|\right)+2 \lambda_{n}\left\|A x_{n}-A p\right\|\left\|u_{n}-p\right\| .
\end{aligned}
$$


Then we get

$$
\begin{aligned}
g\left(\left\|x_{n}-u_{n}\right\|\right) \leq & \left\|x_{n}-p\right\|^{2}-\left\|x_{n+1}-p\right\|^{2}+\alpha_{n}\left\|f\left(x_{n}\right)-p\right\|^{2}+2 \lambda_{n}\left\|A x_{n}-A p\right\|\left\|u_{n}-p\right\| \\
\leq & \left\|x_{n}-x_{n+1}\right\|\left(\left\|x_{n}-p\right\|+\left\|x_{n+1}-p\right\|\right)+\alpha_{n}\left\|f\left(x_{n}\right)-p\right\|^{2} \\
& +2 \lambda_{n}\left\|A x_{n}-A p\right\|\left\|u_{n}-p\right\| .
\end{aligned}
$$

By (C1), (3.10), and (3.13), we have

$$
\lim _{n \rightarrow \infty} g\left(\left\|x_{n}-u_{n}\right\|\right)=0
$$

It follows from the property of $g$ that

$$
\lim _{n \rightarrow \infty}\left\|x_{n}-u_{n}\right\|=0
$$

Again, we consider

$$
\begin{aligned}
\left\|y_{n}-p\right\|^{2} & =\left\|Q_{C}\left[\alpha_{n} f\left(x_{n}\right)+\left(1-\alpha_{n}\right) u_{n}\right]-Q_{C} p\right\|^{2} \\
& \leq\left\langle\alpha_{n}\left(f\left(x_{n}\right)-p\right)+\left(1-\alpha_{n}\right)\left(u_{n}-p\right), J\left(y_{n}-p\right)\right\rangle \\
& =\alpha_{n}\left\langle f\left(x_{n}\right)-p, J\left(y_{n}-p\right)\right\rangle+\left(1-\alpha_{n}\right)\left\langle u_{n}-p, J\left(y_{n}-p\right)\right\rangle \\
& \leq \alpha_{n}\left\|f\left(x_{n}\right)-p\right\|\left\|y_{n}-p\right\|+\frac{1}{2}\left[\left\|u_{n}-p\right\|^{2}+\left\|y_{n}-p\right\|^{2}-g\left(\left\|u_{n}-y_{n}\right\|\right)\right] \\
& \leq \alpha_{n}\left\|f\left(x_{n}\right)-p\right\|\left\|y_{n}-p\right\|+\frac{1}{2}\left[\left\|x_{n}-p\right\|^{2}+\left\|y_{n}-p\right\|^{2}-g\left(\left\|u_{n}-y_{n}\right\|\right)\right] .
\end{aligned}
$$

It follows that

$$
\left\|y_{n}-p\right\|^{2} \leq 2 \alpha_{n}\left\|f\left(x_{n}\right)-p\right\|\left\|y_{n}-p\right\|+\left\|x_{n}-p\right\|^{2}-g\left(\left\|u_{n}-y_{n}\right\|\right) .
$$

By using (3.11), we obtain

$$
\begin{aligned}
\left\|x_{n+1}-p\right\|^{2} \leq & \beta_{n}\left\|x_{n}-p\right\|^{2}+\left(1-\beta_{n}\right)\left[\alpha_{n}\left\|f\left(x_{n}\right)-p\right\|^{2}+\left(1-\alpha_{n}\right)\left\|y_{n}-p\right\|^{2}\right] \\
= & \beta_{n}\left\|x_{n}-p\right\|^{2}+\alpha_{n}\left(1-\beta_{n}\right)\left\|f\left(x_{n}\right)-p\right\|^{2}+\left(1-\alpha_{n}\right)\left(1-\beta_{n}\right)\left\|y_{n}-p\right\|^{2} \\
\leq & \beta_{n}\left\|x_{n}-p\right\|^{2}+\alpha_{n}\left(1-\beta_{n}\right)\left\|f\left(x_{n}\right)-p\right\|^{2} \\
& +\left(1-\alpha_{n}\right)\left(1-\beta_{n}\right)\left[2 \alpha_{n}\left\|f\left(x_{n}\right)-p\right\|\left\|y_{n}-p\right\|+\left\|x_{n}-p\right\|^{2}-g\left(\left\|u_{n}-y_{n}\right\|\right)\right] \\
\leq & \left\|x_{n}-p\right\|^{2}+\alpha_{n}\left\|f\left(x_{n}\right)-p\right\|^{2}+2 \alpha_{n}\left\|f\left(x_{n}\right)-p\right\|\left\|y_{n}-p\right\|-g\left(\left\|u_{n}-y_{n}\right\|\right) .
\end{aligned}
$$


Therefore, we have

$$
\begin{aligned}
g\left(\left\|u_{n}-y_{n}\right\|\right) \leq & \left\|x_{n}-p\right\|^{2}-\left\|x_{n+1}-p\right\|^{2}+\alpha_{n}\left\|f\left(x_{n}\right)-p\right\|^{2}+2 \alpha_{n}\left\|f\left(x_{n}\right)-p\right\|\left\|y_{n}-p\right\| \\
\leq & \left\|x_{n}-x_{n+1}\right\|\left(\left\|x_{n}-p\right\|+\left\|x_{n+1}-p\right\|\right)+2 \alpha_{n}\left\|f\left(x_{n}\right)-p\right\|^{2} \\
& +2 \alpha_{n}\left\|f\left(x_{n}\right)-u_{n}\right\|\left\|y_{n}-u_{n}\right\| .
\end{aligned}
$$

By (C1) and (3.10), we have

$$
\lim _{n \rightarrow \infty} g\left(\left\|u_{n}-y_{n}\right\|\right)=0
$$

From the property of $g$, we get

$$
\lim _{n \rightarrow \infty}\left\|u_{n}-y_{n}\right\|=0
$$

According (3.19) and (3.25), we also have

$$
\lim _{n \rightarrow \infty}\left\|x_{n}-y_{n}\right\|=0
$$

Since

$$
\begin{aligned}
\left\|x_{n}-T\left(\mu_{n}\right) x_{n}\right\| & \leq\left\|x_{n}-T\left(\mu_{n}\right) y_{n}\right\|+\left\|T\left(\mu_{n}\right) y_{n}-T\left(\mu_{n}\right) x_{n}\right\| \\
& \leq\left\|x_{n}-T\left(\mu_{n}\right) y_{n}\right\|+\left\|y_{n}-x_{n}\right\|,
\end{aligned}
$$

from (3.9) and (3.26), we get

$$
\lim _{n \rightarrow \infty}\left\|T\left(\mu_{n}\right) x_{n}-x_{n}\right\|=0
$$

Now, we show that $z \in F:=F(\mathcal{S}) \cap V I(C, A)$. We can choose a sequence $\left\{x_{n_{k}}\right\}$ of $\left\{x_{n}\right\}$ such that $\left\{x_{n_{k}}\right\}$ is bounded, and there exists a subsequence $\left\{x_{n_{k_{j}}}\right\}$ of $\left\{x_{n_{k}}\right\}$ which converges weakly to $z$. Without loss of generality, we can assume that $x_{n_{k}} \rightarrow z$.

(I) We show that $z \in V I(C, A)$. From the assumption, we see that control sequence $\left\{\lambda_{n_{k}}\right\}$ is bounded. So, there exists a subsequence $\left\{\lambda_{n_{k_{j}}}\right\}$ that converges to $\lambda_{0}$. We may assume, without loss of generality, that $\lambda_{n_{k}} \rightarrow \lambda_{0}$. Observe that

$$
\begin{aligned}
\left\|Q_{C}\left(x_{n_{k}}-\lambda_{0} A x_{n_{k}}\right)-x_{n_{k}}\right\| & \leq\left\|Q_{C}\left(x_{n_{k}}-\lambda_{0} A x_{n_{k}}\right)-y_{n_{k}}\right\|+\left\|y_{n_{k}}-x_{n_{k}}\right\| \\
& \leq\left\|\left(x_{n_{k}}-\lambda_{0} A x_{n_{k}}\right)-\left(x_{n_{k}}-\lambda_{n_{k}} A x_{n_{k}}\right)\right\|+\left\|y_{n_{k}}-x_{n_{k}}\right\| \\
& \leq M\left\|\lambda_{n_{k}}-\lambda_{0}\right\|+\left\|y_{n_{k}}-x_{n_{k}}\right\|,
\end{aligned}
$$


where $M$ is as appropriate constant such that $M \geq \sup _{n \geq 1}\left\{\left\|A x_{n}\right\|\right\}$. It follows from (3.26) and $\lambda_{n_{k}} \rightarrow \lambda_{0}$ that

$$
\lim _{k \rightarrow \infty}\left\|Q_{C}\left(x_{n_{k}}-\lambda_{0} A x_{n_{k}}\right)-x_{n_{k}}\right\|=0
$$

We know that $Q_{C}\left(I-\lambda_{0} A\right)$ is nonexpansive, and it follows from Lemma 2.3 that $z \in F\left(Q_{C}(I-\right.$ $\left.\left.\lambda_{0} A\right)\right)$. By using Lemma 2.14, we can obtain that $z \in F\left(Q_{C}\left(I-\lambda_{0} A\right)\right)=V I(C, A)$.

(II) Next, we show that $z \in F(\mathcal{S})$. Let $\mu_{n_{k}} \geq 0$ such that

$$
\mu_{n_{k}} \longrightarrow 0, \quad \frac{\left\|T\left(\mu_{n_{k}}\right) x_{n_{k}}-x_{n_{k}}\right\|}{\mu_{n_{k}}} \longrightarrow 0, \quad k \longrightarrow \infty
$$

Fix $t>0$. Notice that

$$
\begin{aligned}
\left\|x_{n_{k}}-T(t) z\right\| \leq & \sum_{i=0}^{\left[t / \mu_{n_{k}}\right]-1}\left\|T\left((i+1) \mu_{n_{k}}\right) x_{n_{k}}-T\left(i \mu_{n_{k}}\right) x_{n_{k}}\right\| \\
& +\left\|T\left(\left[\frac{t}{\mu_{n_{k}}}\right] \mu_{k}\right) x_{n_{k}}-T\left(\left[\frac{t}{\mu_{n_{k}}}\right] \mu_{n_{k}}\right) z\right\|+\left\|T\left(\left[\frac{t}{\mu_{n_{k}}}\right] \mu_{n_{k}}\right) z-T(t) z\right\| \\
\leq & {\left[\frac{t}{\mu_{n_{k}}}\right]\left\|T\left(\mu_{n_{k}}\right) x_{n_{k}}-x_{n_{k}}\right\|+\left\|x_{n_{k}}-z\right\|+\left\|T\left(t-\left[\frac{t}{\mu_{n_{k}}}\right] \mu_{n_{k}}\right) z-z\right\| } \\
\leq & t \frac{\left\|T\left(\mu_{n_{k}}\right) x_{n_{k}}-x_{n_{k}}\right\|}{\mu_{n_{k}}}+\left\|x_{n_{k}}-p\right\|+\left\|T\left(t-\left[\frac{t}{\mu_{n_{k}}}\right] \mu_{n_{k}}\right) z-z\right\| \\
& \leq t \frac{\left\|T\left(\mu_{n_{k}}\right) x_{n_{k}}-x_{n_{k}}\right\|}{\mu_{n_{k}}}+\left\|x_{n_{k}}-p\right\|+\max \left\{\|T(s) z-z\|: 0 \leq s \leq \mu_{n_{k}}\right\} .
\end{aligned}
$$

For all $k \in \mathbb{N}$, we have

$$
\limsup _{k \rightarrow \infty}\left\|x_{n_{k}}-T(t) z\right\| \leq \limsup _{k \rightarrow \infty}\left\|x_{n_{k}}-z\right\|
$$

Since a Banach space $E$ with a weakly sequentially continuous duality mapping satisfies the Opial's condition, this implies that $T(t) z=z$. Therefore $z \in F(\mathcal{S})$, so $z \in F$.

Next, we show that $\lim \sup _{n \rightarrow \infty}\left\langle(f-I) x^{*}, J\left(y_{n}-x^{*}\right)\right\rangle \leq 0$, where $x^{*}=Q_{F} f x^{*}, Q_{F}$ is a sunny nonexpansive retraction of $C$ onto $F$. Since we have (3.26) and $x_{n_{k}} \rightarrow z$, then we have $y_{n_{k}} \rightarrow z$ such that

$$
\begin{aligned}
\limsup _{n \rightarrow \infty}\left\langle(f-I) x^{*}, J\left(y_{n}-x^{*}\right)\right\rangle & =\lim _{k \rightarrow \infty}\left\langle(f-I) x^{*}, J\left(y_{n_{k}}-x^{*}\right)\right\rangle \\
& =\lim _{k \rightarrow \infty}\left\langle(f-I) x^{*}, J\left(z-x^{*}\right)\right\rangle \leq 0 .
\end{aligned}
$$


Finally, we show that $\left\{x_{n}\right\}$ converges strongly to $x^{*}$. Suppose that $\left\{x_{n}\right\}$ does not converge strongly to $x^{*}$, and then there exist $\epsilon>0$ and a subsequence $\left\{x_{n_{i}}\right\}$ of $\left\{x_{n}\right\}$ such that $\left\|x_{n_{i}}-x^{*}\right\|>\epsilon$ for all $i \in \mathbb{N}$. By Proposition 2.12, for this $\epsilon$ there exists $r \in(0,1)$ such that

$$
\left\|f\left(x_{n_{i}}\right)-f(p)\right\| \leq r\left\|x_{n_{i}}-p\right\| .
$$

So, by Lemma 2.7, we have

$$
\begin{aligned}
\left\|y_{n_{i}}-x^{*}\right\|^{2}= & \left\|Q_{C}\left[\alpha_{n} f\left(x_{n_{i}}\right)+\left(1-\alpha_{n_{i}}\right) u_{n_{i}}\right]-Q_{C} x^{*}\right\|^{2} \\
\leq & \left\langle\alpha_{n_{i}}\left(f\left(x_{n_{i}}\right)-x^{*}\right)+\left(1-\alpha_{n_{i}}\right)\left(u_{n_{i}}-x^{*}\right), J\left(y_{n_{i}}-x^{*}\right)\right\rangle \\
= & \alpha_{n_{i}}\left\langle f\left(x_{n_{i}}\right)-x^{*}, J\left(y_{n_{i}}-x^{*}\right)\right\rangle+\left(1-\alpha_{n_{i}}\right)\left\langle u_{n_{i}}-x^{*}, J\left(y_{n_{i}}-x^{*}\right)\right\rangle \\
\leq & \alpha_{n_{i}}\left\langle f\left(x_{n_{i}}\right)-f\left(x^{*}\right), J\left(y_{n_{i}}-x^{*}\right)\right\rangle+\alpha_{n_{i}}\left\langle f\left(x^{*}\right)-x^{*}, J\left(y_{n_{i}}-x^{*}\right)\right\rangle \\
& +\left(1-\alpha_{n_{i}}\right)\left\|u_{n_{i}}-x^{*}\right\|\left\|y_{n_{i}}-x^{*}\right\| \\
\leq & r \alpha_{n_{i}}\left\|x_{n_{i}}-x^{*}\right\|\left\|y_{n_{i}}-x^{*}\right\|+\alpha_{n_{i}}\left\langle f\left(x^{*}\right)-x^{*}, J\left(y_{n_{i}}-x^{*}\right)\right\rangle \\
& +\left(1-\alpha_{n_{i}}\right)\left\|u_{n_{i}}-x^{*}\right\|\left\|y_{n_{i}}-x^{*}\right\| \\
\leq & r \alpha_{n_{i}}\left\|x_{n_{i}}-x^{*}\right\|^{2}+\alpha_{n_{i}}\left\langle f\left(x^{*}\right)-x^{*}, J\left(y_{n_{i}}-x^{*}\right)\right\rangle+\left(1-\alpha_{n_{i}}\right)\left\|x_{n_{i}}-x^{*}\right\|^{2} \\
= & \left(1-(1-r) \alpha_{n_{i}}\right)\left\|x_{n_{i}}-x^{*}\right\|^{2}+\alpha_{n_{i}}\left\langle f\left(x^{*}\right)-x^{*}, J\left(y_{n_{i}}-x^{*}\right)\right\rangle .
\end{aligned}
$$

It follows from (3.11) that

$$
\begin{aligned}
\left\|x_{n_{i}+1}-p\right\|^{2} \leq & \beta_{n_{i}}\left\|x_{n_{i}}-p\right\|^{2}+\left(1-\beta_{n_{i}}\right)\left\|y_{n_{i}}-p\right\|^{2} \\
\leq & \beta_{n_{i}}\left\|x_{n_{i}}-p\right\|^{2}+\left(1-\beta_{n_{i}}\right) \\
& \times\left[\left(1-(1-r) \alpha_{n_{i}}\right)\left\|x_{n_{i}}-x^{*}\right\|^{2}+\alpha_{n_{i}}\left\langle f\left(x^{*}\right)-x^{*}, J\left(y_{n_{i}}-x^{*}\right)\right\rangle\right] \\
= & {\left[1-(1-r)\left(1-\beta_{n_{i}}\right) \alpha_{n_{i}}\right]\left\|x_{n_{i}}-x^{*}\right\|^{2}+\left(1-\beta_{n_{i}}\right) \alpha_{n_{i}}\left\langle f\left(x^{*}\right)-x^{*}, J\left(y_{n_{i}}-x^{*}\right)\right\rangle . }
\end{aligned}
$$

Now, from (C1), (3.34) and applying Lemma 2.9 to (3.37), we get $\left\|x_{n}-x^{*}\right\| \rightarrow 0$ as $n \rightarrow \infty$. This is a contradiction, and hence the sequence $\left\{x_{n}\right\}$ converges strongly to $x^{*} \in F$. The proof is completed.

Corollary 3.2. Let E be a uniformly convex and 2-uniformly smooth Banach space with the best smooth constant $K$ and $C$ a nonempty closed convex subset of $E$. Let $Q_{C}$ be a sunny nonexpansive retraction from $E$ onto $C$ and $A: C \rightarrow E$ be an $\beta$-inverse strongly accretive operator. Let $\mathcal{S}=\{T(t)$ : $t \geq 0\}$ be a nonexpansive semigroup from $C$ into it self and $f$ be a Meir-Keeler contraction of $C$ into 
itself. Suppose that $F:=F(S) \cap V I(C, A) \neq \emptyset$. For arbitrary given $x_{1} \in C$, the sequences $\left\{x_{n}\right\}$ are generated by

$$
\begin{gathered}
u_{n}=Q_{C}\left(x_{n}-\lambda_{n} A x_{n}\right), \\
y_{n}=Q_{C}\left[\alpha_{n} f\left(x_{n}\right)+\left(1-\alpha_{n}\right) u_{n}\right], \\
x_{n+1}=\beta_{n} x_{n}+\left(1-\beta_{n}\right) \frac{1}{t_{n}} \int_{0}^{t_{n}} T(s) y_{n} d s, \quad \forall n \geq 1,
\end{gathered}
$$

where $\left\{\alpha_{n}\right\},\left\{\beta_{n}\right\} \subset(0,1)$, and $\left\{\lambda_{n}\right\} \subset(0, \infty)$ satisfy the conditions (C1)-(C3) in Theorem 3.1 and assume that $\lim _{n \rightarrow \infty} \sup _{x \in \tilde{C}}\left\|\left(1 / t_{n+1}\right) \int_{0}^{t_{n+1}} T(s) x d s-\left(1 / t_{n}\right) \int_{0}^{t_{n}} T(s) x d s\right\|=0, \tilde{C}$ bounded subset of $C, \lim _{n \rightarrow \infty} \mu_{n}=\infty$ and $\lim _{n \rightarrow \infty}\left(\mu_{n} / \mu_{n+1}\right)=1$. Then $\left\{x_{n}\right\}$ converges strongly to $x^{*}=Q_{F} f x^{*} \in F$, which also solves the following variational inequality:

$$
\left\langle(f-I) x^{*}, J\left(z-x^{*}\right)\right\rangle \leq 0, \quad \forall z \in F .
$$

Corollary 3.3. Let E be a uniformly convex and 2-uniformly smooth Banach space with the best smooth constant $K$ and $C$ a nonempty closed convex subset of $E$. Let $Q_{C}$ be a sunny nonexpansive retraction from $E$ onto $C$. Let $S=\{T(t): t \geq 0\}$ be a nonexpansive semigroup from $C$ into itself and $f$ be a Meir-Keeler contraction of $C$ into itself. Suppose that $F(S) \neq \emptyset,\left\{\alpha_{n}\right\},\left\{\beta_{n}\right\} \subset(0,1)$, and $\left\{\mu_{n}\right\} \subset(0, \infty)$ satisfy the conditions (C1), (C2), (C4), and (C5) in Theorem 3.1. For arbitrary given $x_{1} \in C$, the sequences $\left\{x_{n}\right\}$ are generated by

$$
\begin{gathered}
y_{n}=Q_{C}\left[\alpha_{n} f\left(x_{n}\right)+\left(1-\alpha_{n}\right) x_{n}\right], \\
x_{n+1}=\beta_{n} x_{n}+\left(1-\beta_{n}\right) T\left(\mu_{n}\right) y_{n}, \quad \forall n \geq 1 .
\end{gathered}
$$

Then $\left\{x_{n}\right\}$ converges strongly to $x^{*}=Q_{F} f x^{*} \in F(\mathcal{S})$, which also solves the following variational inequality:

$$
\left\langle(f-I) x^{*}, J\left(z-x^{*}\right)\right\rangle \leq 0, \quad \forall z \in F .
$$

Proof. Taking $A=0$ in Theorem 3.1, we can conclude the desired conclusion easily. This completes the proof.

Corollary 3.4. Let E be a uniformly convex and 2-uniformly smooth Banach space with the best smooth constant $K$ and $C$ a nonempty closed convex subset of $E$. Let $Q_{C}$ be a sunny nonexpansive retraction from $E$ onto $C$ and $A: C \rightarrow E$ be an $\beta$-inverse strongly accretive operator. Let $f$ be a Meir-Keeler contraction of $C$ into itself. Suppose that $F:=V I(C, A) \neq \emptyset,\left\{\alpha_{n}\right\},\left\{\beta_{n}\right\} \subset(0,1)$ and $\left\{\lambda_{n}\right\} \subset(0, \infty)$ satisfy the conditions (C1)-(C3) in Theorem 3.1. For arbitrary given $x_{1} \in C$, the sequences $\left\{x_{n}\right\}$ are generated by

$$
\begin{gathered}
u_{n}=Q_{C}\left(x_{n}-\lambda_{n} A x_{n}\right), \\
y_{n}=Q_{C}\left[\alpha_{n} f\left(x_{n}\right)+\left(1-\alpha_{n}\right) u_{n}\right], \\
x_{n+1}=\beta_{n} x_{n}+\left(1-\beta_{n}\right) y_{n}, \quad \forall n \geq 1 .
\end{gathered}
$$


Then $\left\{x_{n}\right\}$ converges strongly to $x^{*}=Q_{F} f x^{*} \in F$, which also solves the following variational inequality:

$$
\left\langle(f-I) x^{*}, J\left(z-x^{*}\right)\right\rangle \leq 0, \quad \forall z \in F .
$$

Proof. Taking $\mu_{n}=0$ for all $n \geq 1$ in Theorem 3.1, we can conclude the desired conclusion easily. This completes the proof.

Corollary 3.5. Let E be a uniformly convex and 2-uniformly smooth Banach space with the best smooth constant $K$ and $C$ a nonempty closed convex subset of $E$. Let $Q_{C}$ be a sunny nonexpansive retraction from $E$ onto $C$ and $A: C \rightarrow E$ be an $\beta$ strongly accretive and L-Lipschitz continuous operator. Let $\mathcal{S}=\{T(t): t \geq 0\}$ be a nonexpansive semigroup from $C$ into it self and $f$ be a MeirKeeler contraction of $C$ into itself. Suppose that $F:=F(S) \cap V I(C, A) \neq \emptyset,\left\{\alpha_{n}\right\},\left\{\beta_{n}\right\} \subset(0,1)$ and $\left\{\mu_{n}\right\},\left\{\lambda_{n}\right\} \subset(0, \infty)$ satisfy the conditions (C1) and (C3)-(C5) in Theorem 3.1. If the sequence $\left\{x_{n}\right\}$ is generated by $x_{1} \in C$ and (3.1) and $\lim _{n \rightarrow \infty}\left(\lambda_{n+1}-\lambda_{n}\right)=0$ and $0<a \leq \lambda_{n} \leq b<\beta / K^{2} L^{2}$, then the sequence $\left\{x_{n}\right\}$ converges strongly to $x^{*}=Q_{F} f x^{*} \in F$, which also solves the following variational inequality:

$$
\left\langle(f-I) x^{*}, J\left(z-x^{*}\right)\right\rangle \leq 0, \quad \forall z \in F .
$$

Proof. Since $A$ be an $\beta$ strongly accretive and $L$-Lipschitz continuous operator of $C$ into $E$, we have

$$
\langle A x-A y, j(x-y)\rangle \geq \beta\|x-y\|^{2} \geq \frac{\beta}{L^{2}}\|A x-A y\|^{2}, \quad \forall x, y \in C .
$$

Therefore, $A$ is $\left(\beta / L^{2}\right)$-inverse strongly accretive. Using Theorem 3.1, we can obtain that $\left\{x_{n}\right\}$ converges strongly to $x^{*}$. This completes the proof.

The following corollary is defined in a real Hilbert space. Let $C$ be a closed convex subset of a real Hilbert space $H$. Let $A: C \rightarrow H$ be a mapping. The classical variational inequality problems are to find $x \in C$ such that

$$
\langle A x, y-x\rangle \geq 0
$$

for all $y \in C$. For every point $x \in H$, there exists a unique nearest point in $C$, denoted by $P_{C} x$, such that

$$
\left\|x-P_{C} x\right\| \leq\|x-y\|, \quad \forall y \in C
$$

$P_{C}$ is called the metric projection of $H$ onto $C$. It is well known that $P_{C}$ is a nonexpansive mapping of $H$ onto $C$ and satisfies

$$
\left\langle x-y, P_{C} x-P_{C} y\right\rangle \geq\left\|P_{C} x-P_{C} y\right\|^{2},
$$


for every $x, y \in H$. Moreover, $P_{C} x$ is characterized by the following properties: $P_{C} x \in C$ and

$$
\begin{gathered}
\left\langle x-P_{C} x, y-P_{C} x\right\rangle \leq 0 \\
\|x-y\|^{2} \geq\left\|x-P_{C} x\right\|^{2}+\left\|y-P_{C} x\right\|^{2},
\end{gathered}
$$

for all $x \in H, y \in C$.

It is well known in Hilbert spaces the smooth constant $K=\sqrt{2} / 2$ and $J=I$ (identity mapping). From Theorem 3.1, we can obtain the following result immediately.

Corollary 3.6. Let $C$ be a nonempty compact convex subset of a real Hilbert space $H$. Let $P_{C}$ be a metric projection of $H$ onto $C$ and $A: C \rightarrow E$ be an $\beta$-inverse strongly accretive operator. Let $\mathcal{S}=\{T(t): t \geq 0\}$ be a nonexpansive semigroup from $C$ into itself and $f$ be a Meir-Keeler contraction of $C$ into itself. Suppose that $F:=F(\mathcal{S}) \cap V I(C, A) \neq \emptyset,\left\{\alpha_{n}\right\},\left\{\beta_{n}\right\} \subset(0,1)$ and $\left\{\mu_{n}\right\},\left\{\lambda_{n}\right\} \subset(0, \infty)$ satisfy the conditions (C1)-(C5) in Theorem 3.1. For arbitrary given $x_{1} \in C$, the sequences $\left\{x_{n}\right\}$ are generated by

$$
\begin{gathered}
u_{n}=P_{C}\left(x_{n}-\lambda_{n} A x_{n}\right), \\
y_{n}=P_{C}\left[\alpha_{n} f\left(x_{n}\right)+\left(1-\alpha_{n}\right) u_{n}\right], \\
x_{n+1}=\beta_{n} x_{n}+\left(1-\beta_{n}\right) T\left(\mu_{n}\right) y_{n}, \quad \forall n \geq 1 .
\end{gathered}
$$

Then $\left\{x_{n}\right\}$ converges strongly to $x^{*}=P_{F} f x^{*} \in F$ which also solves the following variational inequality:

$$
\left\langle(f-I) x^{*}, z-x^{*}\right\rangle \leq 0, \quad \forall z \in F
$$

Remark 3.7. Question and Open problems. Can we extend Theorem 3.1 to more general variational inequalities in the sense of Noor [1] on Banach spaces?

\section{Acknowledgments}

The first author gratefully acknowledges support provided by King Mongkuts University of Technology Thonburi (KMUTT) during the first authors stay at King Mongkuts University of Technology Thonburi (KMUTT) as a postdoctoral fellow. Moreover, the authors would like to thank the Higher Education Research Promotion and National Research University Project of Thailand, Office of the Higher Education Commission (NRU-CSEC no. 55000613) for financial support. Both authors thank the referees for their comments which improved the presentation of this paper.

\section{References}

[1] M. A. Noor, "General variational inequalities," Applied Mathematics Letters, vol. 1, no. 2, pp. 119-122, 1988.

[2] M. A. Noor, "Some developments in general variational inequalities," Applied Mathematics and Computation, vol. 152, no. 1, pp. 199-277, 2004. 
[3] M. A Noor, On variational inequalities [Ph.D. thesis], Brunel University, London, UK, 1975.

[4] M. A. Noor, "Some iterative techniques for general monotone variational inequalities," Optimization, vol. 46, no. 4, pp. 391-401, 1999.

[5] M. A. Noor, "New approximation schemes for general variational inequalities," Journal of Mathematical Analysis and Applications, vol. 251, no. 1, pp. 217-229, 2000.

[6] M. A. Noor and T. M. Rassias, "A class of projection methods for general variational inequalities," Journal of Mathematical Analysis and Applications, vol. 268, no. 1, pp. 334-343, 2002.

[7] M. A. Noor, K. I. Noor, and T. M. Rassias, "Some aspects of variational inequalities," Journal of Computational and Applied Mathematics, vol. 47, no. 3, pp. 285-312, 1993.

[8] Y. Yao and S. Maruster, "Strong convergence of an iterative algorithm for variational inequalities in Banach spaces," Mathematical and Computer Modelling, vol. 54, no. 1-2, pp. 325-329, 2011.

[9] A. T. M. Lau, "Amenability and fixed point property for semigroup of nonexpansive mappings," in Fixed Point Theory and Applications, M. A. Thera and J. B. Baillon, Eds., vol. 252 of Pitman Research Notes in Mathematics Series, pp. 303-313, Longman Scientific \& Technical, Harlow, UK, 1991.

[10] A. T.-M. Lau and Y. Zhang, "Fixed point properties od semigroups of non-expansive mappings," Journal of Functional Analysis, vol. 245, pp. 2534-2554, 2008.

[11] K. Aoyama, H. Iiduka, and W. Takahashi, "Weak convergence of an iterative sequence for accretive operators in Banach spaces," Fixed Point Theory and Applications, Article ID 35390, 13 pages, 2006.

[12] X. N. Li and J. S. Gu, "Strong convergence of modified Ishikawa iteration for a nonexpansive semigroup in Banach spaces," Nonlinear Analysis A, vol. 73, no. 4, pp. 1085-1092, 2010.

[13] R. Wangkeeree and P. Preechasilp, "Modified noor iterations for nonexpansive semigroups with generalized contraction in Banach spaces," Journal of Inequalities and Applications, vol. 2012, p. 6, 2012.

[14] J.-P. Gossez and E. Lami Dozo, "Some geometric properties related to the fixed point theory for nonexpansive mappings," Pacific Journal of Mathematics, vol. 40, pp. 565-573, 1972.

[15] S. Reich, "Asymptotic behavior of contractions in Banach spaces," Journal of Mathematical Analysis and Applications, vol. 44, no. 1, pp. 57-70, 1973.

[16] S. Kitahara and W. Takahashi, "Image recovery by convex combinations of sunny nonexpansive retractions," Topological Methods in Nonlinear Analysis, vol. 2, no. 2, pp. 333-342, 1993.

[17] F.E. Browder, "Nonlinear operators and nonlinear equations of evolution in Banach space," Proceedings of Symposia in Pure Mathematics, vol. 18, pp. 78-81, 1976.

[18] H. K. Xu, "Inequalities in Banach spaces with applications," Nonlinear Analysis A, vol. 16, no. 12, pp. 1127-1138, 1991.

[19] S. Kamimura and W. Takahashi, "Strong convergence of a proximal-type algorithm in a Banach space," SIAM Journal on Optimization, vol. 13, no. 3, pp. 938-945, 2002.

[20] L. S. Liu, "Ishikawa and Mann iterative process with errors for nonlinear strongly accretive mappings in Banach spaces," Journal of Mathematical Analysis and Applications, vol. 194, no. 1, pp. 114-125, 1995.

[21] T. Suzuki, "Strong convergence of Krasnoselskii and Mann's type sequences for one-parameter nonexpansive semigroups without Bochner integrals," Journal of Mathematical Analysis and Applications, vol. 305, no. 1, pp. 227-239, 2005.

[22] H.-K. Xu, "Viscosity approximation methods for nonexpansive mappings," Journal of Mathematical Analysis and Applications, vol. 298, no. 1, pp. 279-291, 2004.

[23] A. Meir and E. Keeler, "A theorem on contraction mappings," Journal of Mathematical Analysis and Applications, vol. 28, pp. 326-329, 1969.

[24] T.-C. Lim, "On characterizations of Meir-Keeler contractive maps," Nonlinear Analysis A, vol. 46, no. 1, pp. 113-120, 2001. 


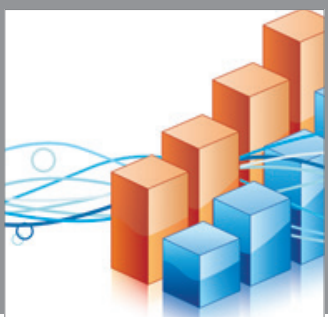

Advances in

Operations Research

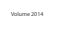

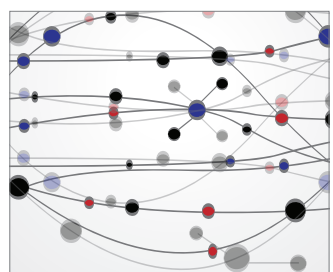

\section{The Scientific} World Journal
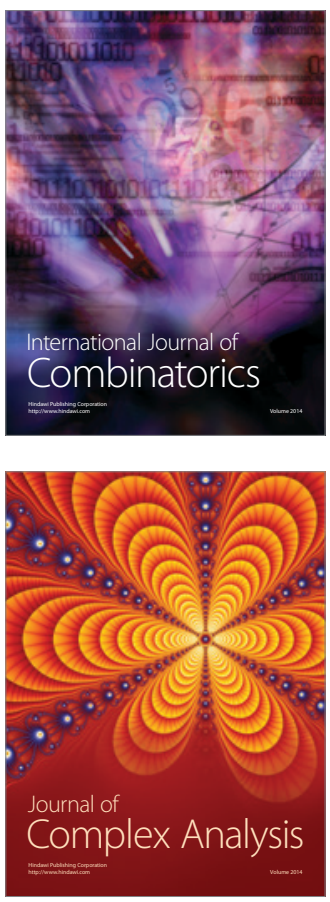

International Journal of

Mathematics and

Mathematical

Sciences
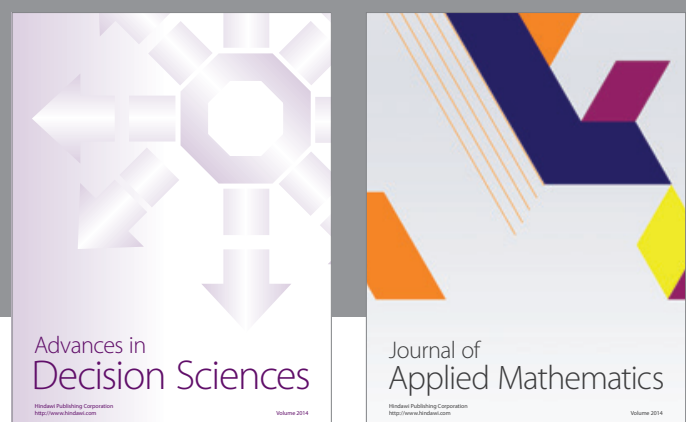

Journal of

Applied Mathematics
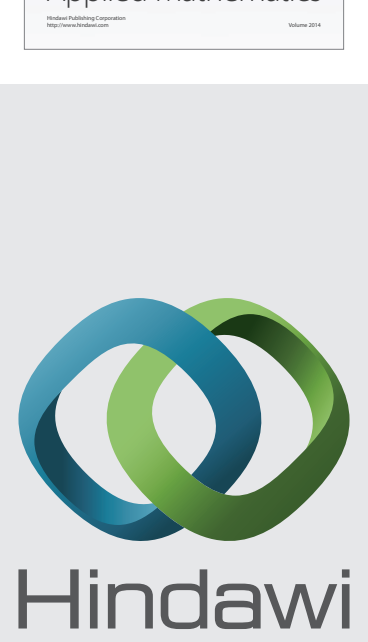

Submit your manuscripts at http://www.hindawi.com
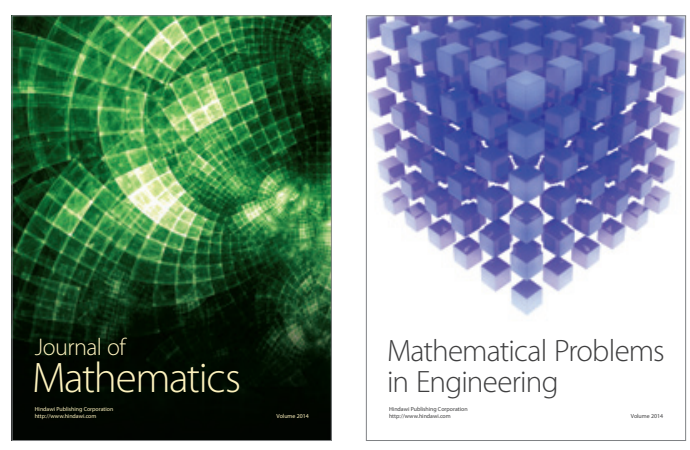

Mathematical Problems in Engineering
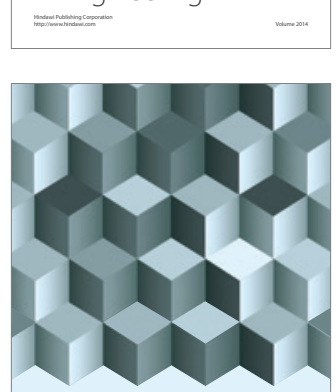

Journal of

Function Spaces
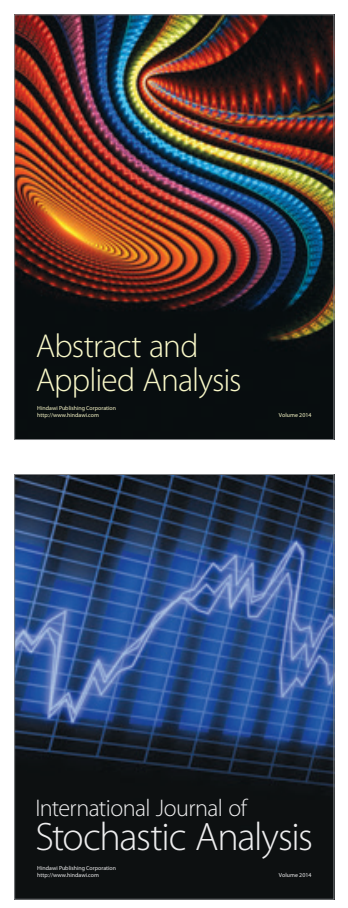

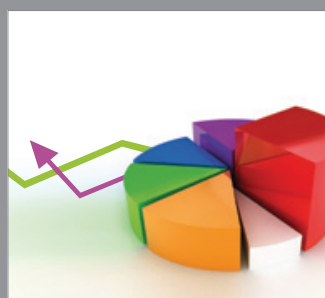

ournal of

Probability and Statistics

Promensencen
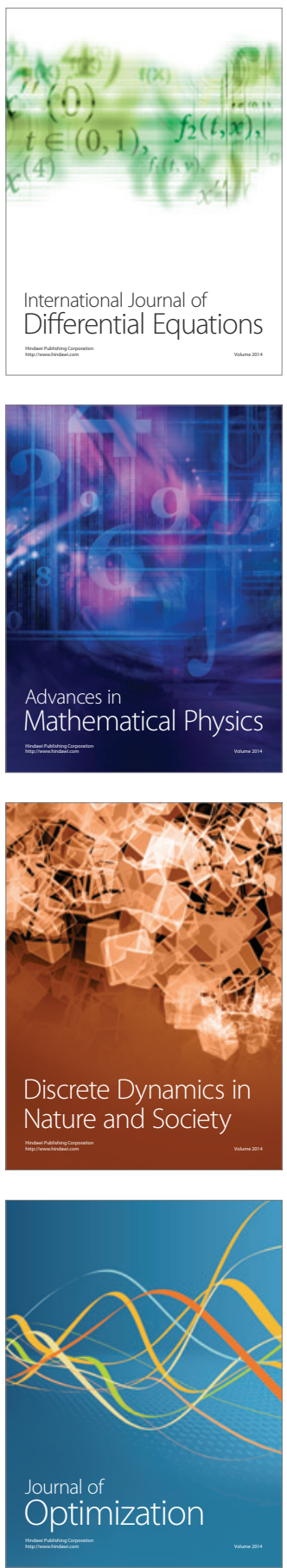